\title{
Perfil De Enojo En Estudiantes De Tres Universidades CUMex. Un Estudio Comparativo
}

Norma Angélica Ortega Andrade, Dra.

Ana María Rivera Guerrero, Mtra.

Universidad Autónoma del Estado de Hidalgo, Mexico

María de los Dolores Valadez, Dra.

Universidad de Guadalajara, Mexico

Claudia Adriana Calvillo Rios, Mtra.

Universidad Autónoma de Zacatecas, Mexico

doi: 10.19044/esj.2017.v13n20p112 URL:http://dx.doi.org/10.19044/esj.2017.v13n20p112

\begin{abstract}
The present study aimed to obtain a profile of the anger in students of the degree in psychology of three universities participating in the Consortium of Mexican Universities (CUMex), through a descriptive-comparative study. 300 students participated in the bachelor's degree in psychology. The research design was non-experimental cross-sectional. Descriptive statistical analyzes of the total sample were carried out, as well as the analysis of variance of a single classification to know if there were significant differences in the anger among the students of the three universities. The results show that in general, the participating population of the three universities is within an average range in this emotion. Regarding the results of the descriptive statistical analysis of the total sample and an analysis of variance of a single classification, statistically significant differences were observed when comparing the three universities, and these differences were found between Guadalajara and Zacatecas; although on average, the students of the three universities score within a normal range of anger. It is possible that these results are natural in students of psychology, which favors the profile of training and its future professional practice.
\end{abstract}

Keywords: Emotion, anger, psychology students, emotional profile

\section{Resumen}

El presente estudio tuvo como objetivo obtener un perfil del enojo en estudiantes de la licenciatura en psicología de tres universidades participantes en el Consorcio de Universidades Mexicanas (CUMex), mediante un estudio descriptivo-comparativo. Participaron 300 estudiantes 
de la licenciatura en psicología. El diseño de investigación fue no experimental transversal. Se llevaron a cabo análisis estadísticos descriptivos de la muestra total, así como el análisis de varianza de una sola clasificación para conocer si existían diferencias significas en cuanto al enojo entre los estudiantes de las tres universidades. Los resultados evidencian que en general, la población participante de las tres universidades se encuentra dentro de un rango promedio en esta emoción. Respecto del resultado del análisis estadístico descriptivo de la muestra total y un análisis de varianza de una sola clasificación, se observaron diferencias estadísticamente significativas al comparar las tres universidades, encontrándose dichas diferencias entre Guadalajara y Zacatecas; aunque en promedio, los estudiantes de las tres universidades puntúan dentro de un rango normal de enojo. Es posible que estos resultados sean naturales en los estudiantes de psicología, lo cual favorece el perfil de formación y su futuro ejercicio profesional.

Palabras-clave: Emoción, enojo, estudiantes de psicología, perfil emocional

\section{Introducción}

Formar profesionales en la ciencia psicológica, es una responsabilidad que las Instituciones de Nivel Superior asumen con el compromiso que implica dotar a los alumnos de conocimientos, habilidades $\mathrm{y}$ actitudes, que de forma integral les permitan atender y afrontar las demandas de la sociedad. En el caso particular de los estudiantes que ingresan, permanecen y egresan de la licenciatura en psicología, se espera que, al ser una profesión que se ubica dentro de las ciencias sociales y humanidades, así como dentro de las ciencias de la salud, su perfil profesiográfico, los caracterice por las aptitudes y habilidades humanas que les lleven a brindar una atención con calidez y calidad a las personas que confían en sus conocimientos.

De acuerdo con Aragón (2011), al ser la psicología una profesión cuya principal actividad se centra en la intervención sobre la vida de las personas, es necesario que el estudiante desarrolle otro tipo de habilidades y competencias que el currículo escolar le proporciona tradicionalmente. Refiere esta autora, que el profesional de esta disciplina debe poseer otro tipo de cualidades que tienen que ver con características propias de su personalidad, es decir, su forma habitual de comportarse, de pensar y de sentir, ya que influirán de manera directa o indirecta en su relación con las personas que acudan a su servicio profesional.

Los perfiles profesionales sin embargo, de diversos programas de psicología han dejado de lado la respuesta a ¿cuáles son esas cualidades que 
debe poseer el psicólogo para ejercer su profesión y cómo lograr su desarrollo? Roe (2003) afirma que una forma de resolver esto, es considerar el modelo basado en competencias, que en la actualidad ha logrado una aceptación por parte de diversas universidades donde se imparte el programa de la licenciatura en psicología, debido a la creciente preocupación por elevar la calidad del desempeño de los egresados, y las graves repercusiones que un mal desempeño profesional, tiene en campos tan sensibles como la salud (Zanatta \& Yurén, 2012).

Sin embargo, aún es poco lo que se ha estudiado sobre las emociones de los estudiantes de psicología, como para definir las características que permitan desarrollar las estrategias pertinentes en los planes de estudio, y de esta forma, contribuir en el perfil progresivo y el de egreso, incluyendo los aspectos emocionales. Algunos estudios se han dirigido a conocer la personalidad, como el realizado en la Facultad de Estudios Superiores de Iztacala, México, a cargo de Aragón (2011), el cual, tuvo como objetivo describir el perfil de personalidad de 433 estudiantes de Psicología, de $1^{\circ}$ a $8^{\circ}$ semestre. Los resultados obtenidos en el cuestionario 16PF, reportaron que los siguientes factores de expresividad emocional, sociabilidad, confianza, dominancia, autosuficiencia, impulsividad, autoestima e inteligencia, se encontraban dentro de los parámetros normales. Se caracterizaron por ser compasivos, sensibles, emotivos, sinceros y francos. Un resultado que llamó la atención es este estudio, fue que los alumnos durante los primeros tres años de la carrera, se caracterizaron por poseer poco autocontrol, así como, por ser poco maduros y poco estables emocionalmente.

Otro estudio es el efectuado por Fores, Morgado, \& Valenzuela (2014), quienes buscaron el perfil cognitivo, emocional y de afrontamiento espiritual de los estudiantes de primer semestre de la licenciatura en psicología, para documentar y trasladar esos resultados a las innovaciones a nivel curricular y lograr con ello, el desarrollo de las competencias de egreso que se establecen. Es importante señalar, según estos autores, cómo la salud mental de los estudiantes es un tema de interés para las universidades, debido a que puede ser un predictor de éxito académico y profesional, que tiene que ver con la credibilidad y el prestigio de la profesión psicológica. Los resultados del estudio, con base en las pruebas aplicadas (MMPI 2, el Cuestionario de Esquemas de Young YSQ-L2, cuestionario de HoneyAlonso de Estilos de aprendizaje, y la Religious Coping breve), explican que en el caso del perfil psicológico, los estudiantes se encontraron en el rango normal en todas las escalas, tratando de mostrar una imagen positiva, un adecuado apego a los convencionalismos sociales y un equilibrio entre la autoprotección y el autodescubrimiento. 
Respecto de los rasgos clínicos, en la prueba de MMPI-2, se encontró en los estudiantes, estados como la estabilidad, el conformismo consigo mismos, realismo, atención al propio estado de salud, sensibilidad, sociabilidad, tenacidad, responsabilidad, relativo ajuste a su rol psicosexual, adaptabilidad en general a las condiciones del medio, autocontrol, pero con poca iniciativa y con un buen equilibrio en sus actitudes y sus conductas introvertidas y extrovertidas (Fores, Morgado, \& Valenzuela, 2014).

Asimismo, destacan resultados en su perfil emocional, de ser personas con un alto nivel de autosacrificio, lo cual les caracteriza como ser capaces de sacrificar sus propias necesidades a las de los demás, evitando de esta manera sentir culpa y ganar autoestima, logrando con ello mantener una conexión emocional con los demás, pues únicamente son felices cuando los demás lo son.

También, en este estudio, los resultados muestran un menor control para manejar las propias emociones e impulsos, así como poca disciplina para alcanzar las metas, baja tolerancia a la frustración y alta impulsividad, como una característica de los participantes, quienes provenían de familias permisivas.

Por otro lado, el estudio realizado por Villalobos-Pérez (2008), dirigido a investigar las características emocionales de los estudiantes de la escuela de psicología de la Universidad Católica de Costa Rica, destacó la presencia de cinco variables valiosas para la carrera, las cuales son: ansiedad por desempeño social, consecuencias fisiológicas (dolores de cabeza, por ejemplo), variantes emocionales consecuencia de la presión ambiental, reacciones ansiosas indeterminadas y, respuestas conductuales a la ansiedad por aspectos sociales. Las dimensiones encontradas muestran que los estudiantes de psicología presentan ansiedad social, consecuencias fisiológicas de la ansiedad y presión ambiental. De igual forma, destaca que las mujeres son más sensibles a las presiones sociales que activan patrones ansiosos, por lo que podrían presentar variaciones emocionales más fuertes que los hombres.

Por su parte, Rodríguez, Sánchez, Valdivia \& Padilla (2005) investigaron también sobre la obtención de perfiles emocionales de estudiantes de licenciatura en Psicología, empleando la Escala TMMS 24, dicho estudio consideró 208 estudiantes de dos universidades (una pública y otra privada) del área metropolitana de Monterrey, N.L., México. Estos autores encontraron que más del $75 \%$ de los estudiantes alcanzaron una adecuada inteligencia emocional, dado que alcanzaron los rangos 2 y 3 en comprensión y regulación de las emociones; lo que también implica el poder diagnosticar y resolver problemas posteriores, y cuya característica es acorde al perfil que se demanda al estudiante de Psicología. Aunado a ello, 
involucra el compromiso con el diálogo, la generación de ideas, la capacidad de ayuda a otros para enfrentar diversas situaciones, y la compasión. Tales resultados, permitieron a los autores, identificar a los estudiantes que alcanzaron el rango 1 en los factores de inteligencia emocional, para futuras propuestas de llevarlos a un proceso más extenso de evaluación y, asimismo, establecer espacios de desarrollo de habilidades de inteligencia emocional.

Un estudio realizado por Rivera (2014), para analizar las emociones positivas y negativas de estudiantes de ciencias de la salud de una universidad pública en el estado de Hidalgo, y sus habilidades emocionales como recursos regulatorios, reportó que los alumnos de psicología se destacan en sub-habilidades de la expresión emocional (expresar las emociones propias con precisión, al expresar una emoción los otros la notan, el nivel de intensidad emocional) del amor, la felicidad y el enojo, cuyas emociones se caracterizan por ser básicas, positivas (amor y felicidad) y negativa (enojo).

De acuerdo con Lange (2001), los sentimientos negativos (expresados en las emociones negativas) son reprimidos regularmente en automático y en gran escala, ya sea porque se sienten desagradables o porque no son sociables; de igual forma, las emociones negativas (entre las que se encuentra el enojo) suelen considerarse como un concepto más elemental, refiriéndose a un estado emocional que incluye sentimientos que varían de intensidad, desde una leve molestia o irritación, hasta la furia o la ira, acompañándose de una excitación del sistema nervioso autónomo.

Ahora bien, dado que la juventud es un momento significativo en el ciclo de vida humana, donde se inician parte de los proyectos vitales (pareja, familia, profesión y trabajo, entre otros), la consolidación y ejecución de estas metas llevan implícita una fuerte carga emocional y cognitiva para el éxito y afirmación de las mismas; es una etapa que, de acuerdo con Mendoza (2011), debe ser entendida como un producto social, que se ve influenciado en gran medida por el lugar que ocupan los jóvenes dentro de la sociedad y por las relaciones que estos establecen con las instancias sociales donde se desenvuelve.

De igual forma, Mingote y Requena (2008) destacan que el bienestar psicológico del joven, depende de su participación en actividades que ellos consideran deseables y apropiadas dentro del grupo al que pertenecen; su autoestima se ve mejorada cuando éste se siente productivo y es capaz de ejercer un control de los sucesos personales significativos, es decir, aquellos que permiten la identificación con un grupo social y facilitan su colaboración y ayuda, además de interiorizar las normas y valores culturales de forma positiva. En cambio, la percepción de incontrolabilidad y la de impredecibilidad influyen para el desarrollo de trastornos de ansiedad y depresión, así como de la conducta. Estos autores afirman que, los jóvenes 
aunque se caracterizan por una buena salud y vitalidad, ellos también muestran un desarrollo emocional pobre e inmaduro, un predominio de actitudes pasivas y carencia de proyectos vitales satisfactorios, con alto riesgo de aislamiento social, depresión, ansiedad, que a veces intenta resolver de forma errónea a través del consumo de adictivos y de la inmersión de bandas que tienen la misión de suplir las carencias de su familia de origen con ideales equivocados.

Esta condición propia de la etapa juvenil, es la que podría encontrarse también en el estudiante de psicología, por la etapa de desarrollo en que se encuentra; sin embargo, resulta necesario estudiar las características específicas de esta población, de tal forma que se cuente con un perfil emocional del enojo que se incluya dentro de la formación integral que éste recibe en los espacios universitarios.

Es importante señalar que el histórico de los programas de las licenciaturas en psicología, muestra que inicialmente su plan curricular, poco se enfocó a atender las necesidades personales de sus estudiantes. Fue en los años 90, cuando se prestó especial interés en proponer un diseño curricular centrado en el estudiante y en su formación integral, para lo cual, se pensó en una estrategia valoral y de desarrollo personal que coadyuvara a elevar la imagen de la profesión y la calidad de vida del estudiante (Zanatta \& Yurén, 2012).

Lograr esto, implica que el profesional de la psicología realice una introspección y aprenda a regular sus emociones, a fin de ser lo más objetivo posibles en el ejercicio de su profesión; sin embargo, resulta preocupante que los programas académicos de las licenciaturas en psicología no lo consideran como parte de la formación curricular de sus estudiantes. Los programas centran su formación en modelos teóricos, aplicación de técnicas y métodos enfocados a la investigación y al ejercicio propio de la profesión.

Es importante considerar que en las universidades donde se imparten programas en psicología se reflexione respecto de la presencia de las emociones, a medida que se avanza en los programas educativos, por lo cual, se sugiere continuar explorando y ahondando esta área emocional, como parte de los planes curriculares. Por tanto, la presente investigación tuvo como objetivo medir el enojo como parte de un perfil emocional de los estudiantes de la licenciatura en psicología de tres universidades participantes en el Consorcio de Universidades Mexicanas (CUMex), mediante un estudio descriptivo-comparativo. 


\section{Método}

\section{Participantes}

En el estudio participaron 300 estudiantes de la licenciatura en psicología de tres universidades pertenecientes al Consorcio de Universidades Mexicanas (CUMex).

La muestra estuvo conformada por 100 estudiantes de cada una de las universidades participantes: Universidades públicas en los estados de Guadalajara, Zacatecas e Hidalgo. El rango de edad osciló entre 18 y 26 años. El muestreo fue no probabilístico de sujetos tipo.

\section{Tipo de estudio y diseño}

El estudio fue de tipo cuantitativo descriptivo comparativo, con un diseño de investigación no experimental transversal.

\section{Instrumentos}

Se utilizó la Prueba de Alcázar, Deffenbacher \& Byrne (2011), cuyo instrumento mide el grado de facilidad con que una persona se enoja, validada para población mexicana; integrada por 10 afirmaciones que dan respuesta a cómo se siente generalmente la persona, a través de cuatro opciones de respuesta que van de casi nunca a casi siempre.

\section{Procedimiento}

La aplicación del instrumento se llevó a cabo en cada universidad, durante el semestre escolar enero-junio 2015, por parte de las investigadoras que participaron en este estudio. Cada investigadora seleccionó a los estudiantes, de acuerdo con el número propuesto para conformar la muestra. Como parte de los lineamientos éticos, se solicitó el consentimiento informado de los estudiantes. Una vez que se aplicó la prueba, se conformó una base de datos, con el objetivo de realizar los análisis estadísticos pertinentes.

\section{Resultados}

El análisis de datos se realizó a través del Paquete Estadístico para las Ciencias Sociales (SPSS) versión 19. Se llevaron a cabo análisis estadísticos descriptivos de la muestra total y un análisis de varianza de una sola clasificación para conocer si existían diferencias significas en cuanto al enojo entre los estudiantes de las tres universidades.

En cuanto a los resultados descriptivos, los estudiantes de la universidad en Zacatecas, tuvieron una media de $M=18.20, D T=7.35$ en la emoción enojo. Los estudiantes de la universidad en Guadalajara, obtuvieron una media de $M=20.53, D T=5.58$ en esta emoción. De igual forma, los 
estudiantes de la universidad en Hidalgo, tuvieron una media de $M=20.17$, $D T=6.45$ en la misma emoción.

Para el caso del puntaje obtenido en la emoción enojo en el presente estudio, las medias se encuentran dentro de un rango que se le denomina puntaje promedio o puntaje normal, lo cual quiere decir que las reacciones de enojo de los estudiantes de las tres universidades son similares a las de la mayoría de la gente.

Respecto del resultado del análisis de varianza, se observaron datos estadísticamente significativos al comparar las universidades F=3.720 (gl 2) con un nivel de significancia de .05, donde la universidad en Guadalajara y la universidad en Zacatecas presentaron dichas diferencias en cuanto a la emoción enojo con un nivel de significancia de .035.

En cada universidad se identificaron algunos casos de estudiantes que puntuaron por arriba de la media o por debajo de ésta, y con valores extremos respecto del enojo. De acuerdo con Alcazar, Deffenbacher \& Byrne (2011), la evaluación del enojo considera el puntaje máximo de 40 como “alguien que está en mayor riesgo de molestarse fácilmente ante cualquier provocación”, y el puntaje mínimo de 10 para “alguien que es difícil de enojarse”; en la tabla 1, se puede apreciar el número de estudiantes, de cada universidad, que puntuaron por arriba y por debajo de la media.

Contemplando lo anterior, se encontró que, del total de la muestra (de tres universidades) 109 de los participantes caen dentro del rango de 10 a 16 puntos, es decir, un puntaje bajo para el enojo, lo cual representa el 33.3\% de la muestra total. Dentro de este rango, 17 de ellos se encuentran en el extremo bajo, lo cual representa el 5.7\% de los participantes.

Por otro lado, del total de la muestra, 92 participantes caen dentro de los puntajes altos considerando un rango de 23 a 40 puntos, lo que representa el 30.4\%. Sólo 1 caso de éstos tiene un puntaje en el extremo alto para el enojo, lo cual representa el .3\% de los participantes.

Tabla No 1. Resultados de alumnos con puntajes extremos en enojo.

\begin{tabular}{|c|c|c|c|c|}
\hline \multirow{2}{*}{$\begin{array}{c}\text { Universidad } \\
\text { en el estado }\end{array}$} & \multicolumn{2}{|c|}{ Enojo } & \multicolumn{2}{c|}{ Enojo } \\
\cline { 2 - 5 } de: & \multicolumn{2}{|c|}{ Puntaje Alto (23-40) } & \multicolumn{2}{c|}{ Puntaje Bajo (10-16) } \\
\cline { 2 - 5 } & $\begin{array}{c}\text { Extremo Alto } \\
\text { (40-38) }\end{array}$ & Puntajes (36-23) & $\begin{array}{c}\text { Extremo Bajo } \\
(10)\end{array}$ & Puntajes (11-16) \\
\hline Hidalgo & 1 & 33 & 3 & 28 \\
\hline Zacatecas & & 25 & 12 & 39 \\
\hline Guadalajara & \multicolumn{2}{|c|}{92} & 2 & 109 \\
\hline Total & \multicolumn{2}{|c|}{} \\
\hline
\end{tabular}

\section{Discusión y Conclusión}

Los resultados evidencian que en general, la población participante de las tres universidades se encuentra dentro de un rango promedio respecto 
de la emoción enojo; lo cual es de esperarse en los estudiantes de psicología, dado que el perfil de formación así lo requiere, como lo encontró Aragón (2011), en su estudio sobre el perfil de personalidad de universitarios de psicología, quien reportó parámetros normales en el factor de expresividad emocional, afirmando que los estudiantes de psicología deben conceptualizarse como una persona integral, considerando que el desarrollo de sus emociones y las características de su personalidad contribuirán en su desempeño profesional.

De igual forma, lo encontrado en el presente estudio, muestran una similitud con lo reportado por Salas y García (2010) quienes describen las habilidades de inteligencia emocional por carreras universitarias, donde encontraron que tres de las carreras (urbanismo, ingeniería eléctrica y de producción) arrojaron la misma estructura de habilidades emocionales, en quienes se encontró que poseen más habilidades para conservar estados emocionales satisfactorios o cambiar los desagradables, así como para reconocer las emociones distinguidas.

Es importante considerar también que, para la emoción de enojo, al hablar de puntajes promedio o normales encontrados en la población del estudio que nos compete, dicha emoción se encuentra clasificada como una emoción negativa, no obstante, ésta no necesariamente tiene que ser asociada a lo malo o peligroso, siempre y cuando su ocurrencia sea homeostásica (para proveer equilibrio) y parsimoniosa, como lo indican Palmero, Guerrero, Gómez, Carpi y Gorayeb (2011).

Al respecto y en el mismo tenor, Bisquerra (2009) menciona que las emociones negativas no necesariamente son malas, siendo importante no asociar negativo con malo, ya que cuando una persona experimenta dichas emociones tiende a sentirse culpable por establecer esta asociación de manera automática; es inevitable llegar a sentir emociones negativas ante determinados sucesos, y todas las emociones son legítimas y deben ser aceptadas, dado que de éstas puede derivar un comportamiento adaptativo para asegurar la supervivencia o bienestar, como lo es la huida ante algo que produce miedo.

Ahora bien, para el caso de los alumnos que en este estudio puntuaron alto para enojo, y sobre todo el caso extremo alto de entre las tres universidades, el Instituto de Bienestar Subjetivo (ISWB, 2010) deja ver que estas personas están en mayor riesgo de molestarse fácilmente ante cualquier provocación, y son propensos a guardar rencores o reaccionar con enojo; tienden a creer que las cosas suelen ser con intenciones de daño y piensan en la venganza o en el ajuste de cuentas como una respuesta. Algunas de estas personas consideran la agresión física o verbal con la intención de mostrar a los demás cómo se sienten, y el enojo puede durar en ellos por mucho tiempo, ya sea horas o días; ante lo expuesto, es evidente que este tipo de 
personas tiene o genera consecuencias desagradables en los planes de la vida cotidiana personales y de los demás. Al respecto, Bisquerra (2005) deja ver que en un ámbito educativo, es importante que los alumnos cuenten con competencias necesarias para regular sus emociones de forma apropiada, sobre todo en las emociones negativas, como lo es el control del enojo y la tolerancia a la frustración, entre otras competencias.

Considerando esta misma emoción, para los puntajes bajos y extremos bajos que se encontraron en el presente estudio, la ISWB (2010) refiere que es difícil que estas personas se enojen, llegan a controlar su enojo sin guardar rencor y sin explotar, tienden a tomar las cosas con calma, no actúan cuando no han pensado lo que harán; suele ser recomendable que continúen bajo su carácter de tranquilidad; aun cuando la frustración o la emoción de enojo es inevitable en la vida. Cabe señalar que, pudiera ser un tanto de riesgo para la persona el no activarse homesostásicamente, debido al enojo; es decir que la emoción de manera natural provee un equilibrio como se señala anteriormente, en lo planteado por Palmero et al. (2011) y Bisquerra (2009); asimismo, considerando que esta emoción sucede por la valoración de situaciones que se perciben como injustas y que permite utilizar algunos recursos para su afrontamiento (Silverio, 2002).

Por último, es relevante considerar lo que Jiménez y López-Zafra (2009) señalan, respecto de que los estudiantes deben poseer habilidades emocionales que favorezcan su proceso de adaptación académica y social, ya que la labor de lo escolar le requieren utilizar dichas habilidades para facilitar el pensamiento, aumentar su concentración, regular conductas impulsivas y rendir ante situaciones estresantes, las cuales enfrenta durante su trayectoria universitaria. Entonces, si los estudiantes en general, requieren de ello, mucho más los estudiantes de psicología, quienes se enfrentarán a las emociones no sólo como objeto de estudio en su formación, sino que deben ser competentes al regular sus propias emociones; como lo señala Roe (2003) quien menciona a la estabilidad emocional como parte del perfil de competencias que el psicólogo debe poseer para ejercer dicha profesión. Por lo que resulta conveniente, continuar estudiando las emociones de los estudiantes de la licenciatura en psicología, a fin de contar con un perfil emocional, que favorezca el desarrollo de acciones a considerar en regulación emocional, en el transcurso de su formación profesional.

\section{References:}

1. Alcazar, R., Deffenbacher, J. L., \& Byrne, Z. S. (2011). Assessing the factor structure of the anger expression inventory (ML-STAXI) in a Mexican sample. International Journal of Psychology and Psychological Therapy, 11, 307-318. 
2. Aragón, L. (2011). Perfil de personalidad de estudiantes universitarios de la carrera de psicología. El caso de la Facultad de Estudios Superiores Iztacala. Perfiles Educativos, 23(133), 68-87.

3. Bisquerra, R. (2005). La educación emocional en la formación del profesorado. Revista Interuniversitaria de Formación del Profesorado, 19(3), 95-114. Recuperado de http://www.redalyc.org/pdf/274/27411927006.pdf

4. Bisquerra, R. (2009). Psicopedagogía de las emociones. España: Síntesis.

5. Fores, M., Morgado, M., \& Valenzuela, A. (2014). Perfil psicológico, cognitivo y espiritual de estudiantes universitarios de psicología de primer ingreso. Enseñanza e investigación en psicología, 19(2). Recuperado de http://www.redalyc.org/pdf/292/29238007002.pdf

6. Instituto of Subjective Well-Being. (2010). Oxford Happiness Questionnaire. Happiness Formulas. Vancouver: ISWB. Recuperado de http://www.iswb.org/wp-content/uploads/2010/07/happiness-ebook.pdf

7. Jiménez, M. y López-Zafra, E. (2009). Inteligencia emocional y rendimiento escolar: estado actual de la cuestión. Revista Latinoamericana de Psicología, 41(1), 6979. Recuperado de http://www.redalyc.org/pdf/805/80511492005.pdf

8. Lange, S. (2001). El libro de las emociones... luego exísto ( $\left.2^{\mathrm{a}} \mathrm{ed}\right)$. Recuperado de https://books.google.com.mx/books?id=v87VlKUmbzcC\&printsec=f rontcover\&dq=las+emociones\&hl=es\&sa=X\&ei=vN_WUansLJHI9g S4uIDIBQ\#v=onepage \&q=las\%20emociones \&f=false

9. Mendoza, H. (deptiembre/diciembre, 2011). Los estudios sobre la juventud en México. Espiral, Estudios sobre Estado y Sociedad. 18(52), 193-224. Recuperado de http://148.202.18.157/sitios/publicacionesite/pperiod/espiral/espiralp df/espiral52/sociedad1pdf

10. Mingote, C. y Requena, M. (2008). El malestar de los jóvenes. Contextos, raíces y experiencias. Madrid: Díaz de Santos. Recuperado http://books.google.es/books?id=PmYfHk6ps4MC\&pg=PA20\&dq=d esarrollo+emocional+del+joven\&hl=es\&sa $=\mathrm{X} \& \mathrm{ei}=\mathrm{qFwfU} 56 \mathrm{xO} 47 \mathrm{zo}$ ASMolina

11. Palmero, F., Guerrero, C., Gómez, C., Carpi, A. y Gorayeb, R. (2011). Manual de teorías emocionales y motivacionales. Universitat Jaume I. Recuperado de http://www.uji.es/bin/publ/edicions/s57.pdf 
12. Rivera, A. (2014). Emociones y habilidades emocionales como recursos regulatorios en estudiantes de ciencias de la salud (Tesis de maestría). Universidad Autónoma del Estado de Hidalgo, México.

13. Roe, R. (2003). ¿Qué hace competente a un psicólogo? Papeles del psicólogo, 24(86), 1-12. Recuperado de http://www.redalyc.org/pdf/778/77808601.pdf

14. Rodríguez, M., Sánchez, M., Valdivia, J., Padilla, V., (Abril, 2005). Perfil de Inteligencia emocional en estudiantes universitarios. Trabajo presentado en el XXXII Congreso y LXXVI Asamblea del Consejo Nacional para la Enseñanza e Investigación en Psicología, Mexicali, B.C. N.

15. Salas, A. y García, H. (mayo-agosto, 2010). Perfil de inteligencia emocional y carreras universitarias en estudiantes de la Universidad Simón Bolívar. Revista de Ciencias Sociales, 16(2), 226-238. Recuperado de http://www.redalyc.org/pdf/280/28016298004.pdf

16. Silverio, M. (2002). La tristeza: Análisis y propuestas educativas (Tesis Doctoral). Departamento de Psicología Educativa, Evolutiva y Psicobiología, Universidad de La Laguna. Recuperado de ftp://tesis.bbtk.ull.es/ccssyhum/cs124.pdf

17. Villalobos-Pérez, A. (2008). Características emocionales de estudiantes de psicología: un estudio basado en el enfoque de investigación formativa. Avances en Psicología Latinoamericana, 26(2), 252-269. Recuperado de http://www.scielo.org.co/pdf/apl/v26n2/v26n2a11.pdf

18. Zanatta, E. y Yurén, T. (enero-junio, 2012). La formación profesional del psicólogo en México: trayecto de la construcción de su identidad disciplinar. Enseñanza e investigación en psicología, 17(1), 151-170. Recuperado de http://www.redalyc.org/pdf/292/29223246010.pdf 